\title{
ELECTRONIC COMPASS FOR UNMANNED SHIPS
}

\section{ЭЛЕКТРОННЫЙ КОМПАС ДЛЯ АВТОМНЫХ СУДОВ БЕЗ ЭКИПАЖА}

\author{
I.M. Vikulin, DSc, professor, N.S. Mikhailov, PhD student, S.A. Mikhailov, DSc, professor \\ И.М. Викулин, д.ф.-м.н., профессор, Н.С. Михайлов, аспирант, С.А. Михайлов, д.т.н., \\ профессор \\ National University «Odessa Maritime Academy», Ukraine \\ Национальный университет «Одесская морская академия», Украина
}

\begin{abstract}
Principle of action of semiconductor two-collector magnetic transistors and possibility of creation on their basis of electronic compass is covered in the article. Level of automation of marine ships navigation, appearance of the autonomous fully automated ships without a crew, requires presences aboard the ship of device giving out a not visual, but electronic signal about direction of motion. An electronic compass on the basis of magnetic sensor semiconductor elements can decide this task.

There is of most interest an electronic compass, a consisting of semiconductor magnetic sensor element, electric signal on the output of which is proportional to the level of the external magnetic field, and electronic chip processing a signal. In this article the example of laboratory construction of such electronic compass is described and his descriptions over are brought. Important, that this electronic device does not have mobile mechanical parts and mechanisms.

For the increase of sensitiveness of electronic sensor of the magnetic field application of reflector-absorbers of the magnetic field is offered. Ferrite bars are used in this case. Bars are disposed both-side sensor, parallell to optimum direction of the magnetic field. In the experiments it was succeeded to get the increase of sensitiveness of sensors in 400 times. The reflector-absorbers of the magnetic field also allow to improve correlation signal to noise in 100 times.

An electronic compass on the basis of magnetic transistors can be also used and as an element to control the course of marine ship. If a magnetic transistors is set to direction of motion, the change of loading resistances sets the zero of tension between the collectors of magnetic transistor. At deviation of axis from the set direction, tension of one polarity appears between collectors, and in other - opposite. This tension through the system of autopilot can directly control a steering gear and automatically to maintain the set direction of motion of marine ship.

Experimental descriptions over of pre-productions models of magnetic transistors and flows diagrams of compasses are brought. An experiments show that on the basis of two-collector magnetic transistors can be created electronic compass not containing mechanically moving details, that sharply promotes his reliability and durability.

An electronic compass will become the obligatory attribute of future autonomous ships without a crew. He will be able not only to replace a classic magnetic compass but also to allow to realize the span-new functions of ships control.
\end{abstract}

Keywords: safety of navigator, magnetic compass, navigation, unmanned ships, magnetic transistors, reflector-absorbers of the magnetic field, electronic control chip

\section{PЕФЕРАТ}

У роботі розглянутий принцип дії напівпровідникових двохколекторних магнітотранзісторов і можливість створення на їх основі електронного компаса. Рівень автоматизації процесів управління на морських суднах, поява автономних повністю автоматизованих суден без екіпажа, керованих з берега, вимагає наявність на борту судна пристрою, що видає не візуальний, а електронний сигнал про напрям руху. Вирішити це 
завдання може електронний компас на основі магніточутливих напівпровідникових елементів.

Найбільший інтерес представляє електронний компас, що складається 3 напівпровідникового магніточутливого елементу, електричний сигнал на виході якого пропорційний величині зовнішнього магнітного поля, і електронної схеми сигналу. У даній статті описується приклад лабораторної конструкції такого електронного компасу i приводяться його характеристики. Важливо, що даний електронний пристрій не має рухомих механічних частин і механізмів.

Для збільшення чутливості електронного датчика магнітного поля запропоновано застосування концентраторів магнітного поля. У цій якості використовуються феритові стрижні. Стрижні розташовуються з двох сторін датчика, паралельно оптимальному напряму магнітного поля. Посилення індукції концентраторами майже рівне величині їх магнітної проникності. У експериментах вдавалося одержати збільшення чутливості датчиків в 400 разів. Концентратори магнітного поля також дозволяють поліпшити співвідношення сигнал шум в 100 разів.

Електронний компас на основі магнітотранзісторов може також використовуватися і як елемент, що управляє курсом морського судна. Орієнтував магнітотранзістор в заданому напрямі руху, зміною опорів та навантажень, встановлюється нуль напруги між колекторами магнітотранзістора. При відхиленні осі руху від заданого напряму в один бік, між колекторами з'являється напруга однієї полярності, а в іншу - протилежної. Ця напруга через систему авторульового може безпосередньо управляти рульовим механізмом і автоматично витримувати заданий напрям руху морського судна.

Приводяться експериментальні характеристики дослідних зразків магнітотранзісторов і структурні схеми компасів. Експериментально показано, що на основі двохколекторного магнітотранзістора може бути створений електронний компас, що не містить деталей, що механічно переміщаються, що різко підвищує його надійність і міцність.

Електронний компас стане обов'язковим атрибутом майбутніх автономних суден без екіпажа. Він зможе не тільки замінити класичний магнітний компас, але i дозволити реалізувати абсолютно нові функції управління судном.

Ключові слова: безпека судноводіння, магнітний компас, навігація, судна без екіпажу, магнітотранзістор, концентратори магнітного поля, електронна схема управління і контролю

\section{Постановка проблемы в общем виде и ее связь с важными научными или практическими задачами}

Исторически одним из основных рабочих инструментов мореплавателей является магнитный компас, или, как говорят моряки, «компАс». Мореплавателю всегда нужно знать: в каком же направлении идет его судно? Другой основной рабочий инструмент судоводителя - секстан (секстант), устройство для определения своего географического местоположения, уже давно модифицирован и заменен современными техническими средствами. Точность определения места судна с помощью спутниковых радиоэлектронных систем навигации может составлять сегодня доли метра, что вполне удовлетворяет судоводителя. Морской же магнитный компас по-прежнему остается кусочком намагниченного металла на тонкой острой игле. Между тем, все возрастающий уровень автоматизации процессов управления на морских судах, появление в уже самом ближайшем будущем управляемых с берега автономных полностью автоматизированных судов без экипажа, требует наличия на борту судна устройства, выдающего не визуальный, а электронный сигнал о направлении движения. Решить эту задачу может электронный компас на основе магниточувствительных полупроводниковых элементов.

Компас является одним из приборов с широчайшим диапазоном применения от простого наручного компаса до компаса на гражданском судне, военном корабле, самолёте и других подвижных объектах. Главным показателем прибора является его надёжность и 
прочность, которые значительно возрастают при отсутствии в приборе каких-либо механических движущихся деталей.

Анализ последних достижений и публикаций, в которых начато решение данной проблемы и выделение нерешенных ранее частей общей проблемы

В этом плане наибольший интерес представляет электронный компас, состоящий из полупроводникового магниточувствительного элемента, электрический сигнал с которого пропорционален величине внешнего магнитного поля, и электронной схемы, обрабатывающей сигнал. В данной статье описывается пример лабораторной конструкции такого компаса и приводятся его характеристики.

Наибольшей магниточувствительностью из всех полупроводниковых приборов обладает двухколлекторный магнитотранзистор (далее ДМТ), структура которого показана на рис. 1 [1]. ДМТ, например $p-n-p-$ типа, работает следующим образом. При отсутствии магнитного поля $(B=0)$ инжектированные из эмиттера дырки распределяются поровну между коллекторами (сплошные линии на рис.1, а) и их токи равны. В магнитном поле $\otimes B$ (пунктирные линии) поток носителей отклоняется в сторону коллектора $\kappa_{1}$, его ток увеличивается, а ток коллектора $\kappa_{2}$ уменьшается. Кроме эффекта перераспределения носителей между коллекторами, в ДМТ действует также и эффект изменение длины пути носителей. Как видно из рисунка, он состоит в том, что траектория движения носителей, попадающих в $\kappa_{1}$, уменьшается, т.е. сокращается эффективная длина базы, что приводит к дополнительному росту тока. Для коллектора $\kappa_{2}$ этот эффект, наоборот, приводит к дополнительному уменьшению тока. При противоположном направлении магнитного поля $\otimes B$ ток $K_{2}$ растёт, а ток $\kappa_{1}$ уменьшается.

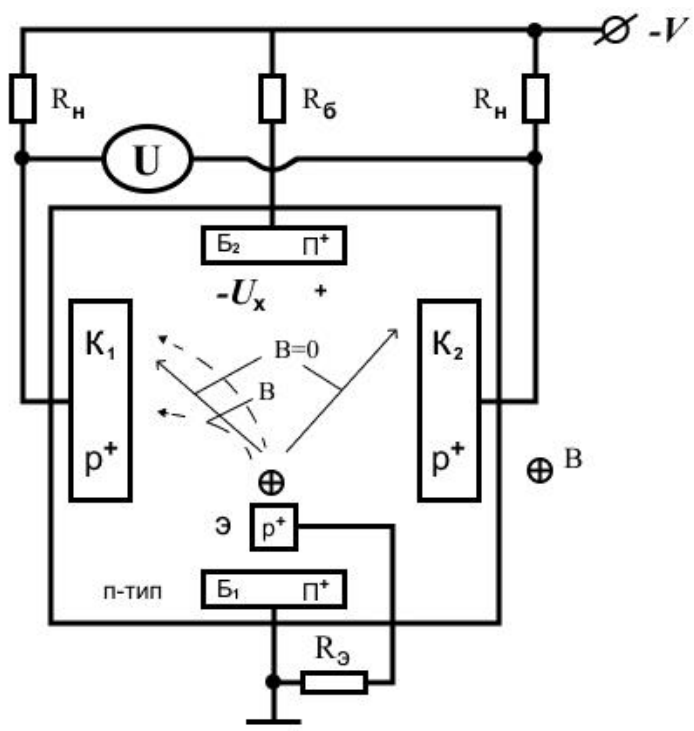

a)

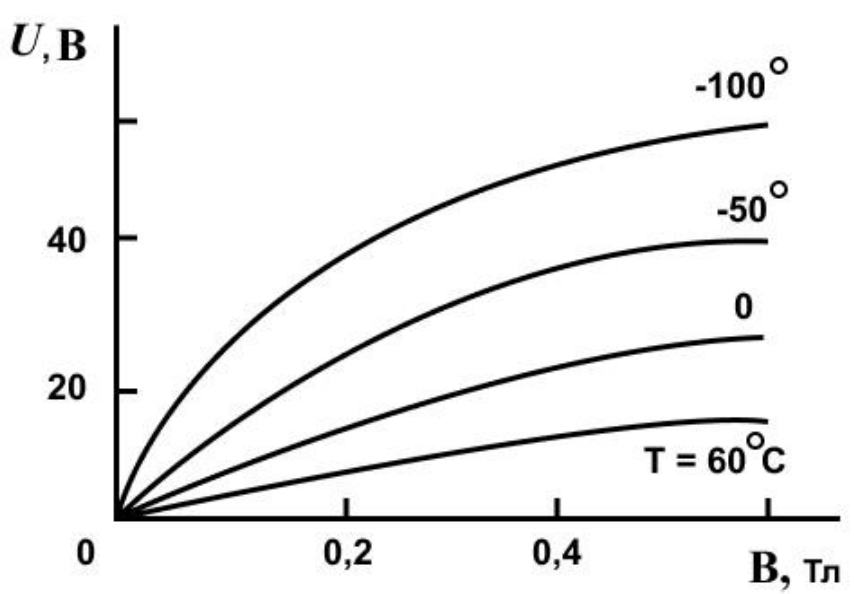

б)

Рис.1.Структура двухколлекторного магнитотранзистора и зависимость напряжения между коллекторами от магнитной индукции при различных температурах

Очевидно, что при $B=0$ в симметричной схеме разность потенциалов между коллекторами $U=0$, с ростом индукции магнитного поля $B$ напряжение $U$ увеличивается. Таким образом, напряжение $U$ является функцией величины магнитного поля, а по его знаку можно определить направление $B$. Как следует из схемы (рис.1), величину $U$ можно определить как: 


$$
U=R_{H}\left(I_{K_{1}}-I_{K_{2}}\right)=R_{H} I_{\ni}\left(h_{21 Б_{1}}-h_{21 Б_{2}}\right)
$$

где $h_{21 Б_{1}}, h_{21 Б_{2}}$ - коэффициенты передачи тока каждой из двух половин ДМТ;

$R_{H}$ - сопротивление нагрузки.

Расчёты показывают [1], что в слабых магнитных полях $(\mu B<<1) h_{21 b_{1}}-h_{21 Б_{2}} \sim B E$, где $E$ - напряженность электрического поля в базе ДМТ между контактами $E_{1}$ и $E_{2}$. Следует отметить, что при протекании тока основных носителей между базовыми контактами $E_{1}$ и $E_{2}$ в магнитном поле в ограниченной базе возникает ЭДС Холла $U_{x}$. Холловское поле отклоняет инжектированные носители в ту же сторону, что есть сила Лоренца. Это увеличивает магниточувствительность ДМТ, но линейность зависимости $U=f(B, E)$ сохраняется, так как $U_{x}$ пропорциональна $B E$.

На рис.1-б приведена типичная зависимость $U(B)$ для кремниевого ДМТ при

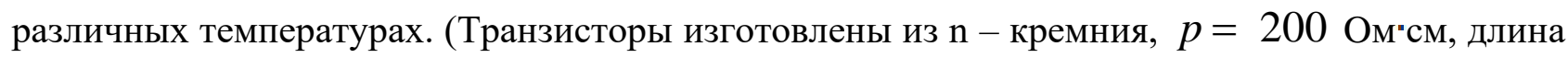
коллекторов 200 мкм, расстояние между ними 150 мкм). При рабочем токе 5 мА магниточувствительность при комнатной температуре составляет около $100 \mathrm{~B} /$ Тл. На рис.2 показана экспериментальная зависимость выходного напряжения ДМТ от угла между направлениями электрического и магнитного поля. Электрическое поле направлено от нижнего базового контакта к верхнему. Вектор магнитного поля вращается в плоскости, перпендикулярной поверхности ДМТ и проходящей через оба базовых контакта. В полном соответствии с принципом действия ДМТ максимальный сигнал наблюдается при $B$, перпендикулярном поверхности ДМТ.

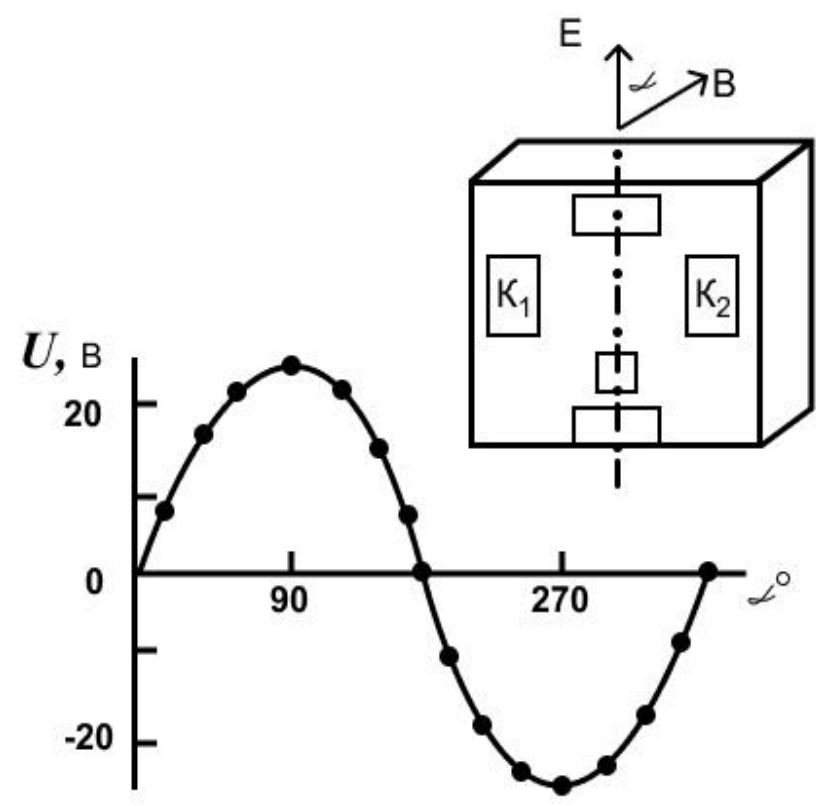

Рис.2. Зависимость выходного напряжения ДМТ от угла между направлением электрического и магнитного полей

\section{Формулировка целей статьи (постановка задачи)}

Целью данной статьи является разработка принципиально нового электронного устройства, регистрирующего магнитное поле Земли и точно указывающее реальное направление по странам света. Данное электронное устройство не имеет подвижных механических частей и механизмов. 


\section{Изложение материала исследования с обоснованием полученных научных результатов}

Увеличить чувствительность датчика магнитного поля на основе ДМТ можно путём применения концентраторов магнитного поля. Обычно в этом качестве используются два ферритовых стержня (МП), длина которых в 40 - 50 раз больше их диаметра. Стержни располагаются с двух сторон датчика, параллельно оптимальному направлению магнитного поля (рис. 3). Концы стержней, примыкающих к ДМТ, заостряются таким образом, чтобы размеры вершины конуса были равны размерам активной части ДМТ. Это позволяет увеличивать концентрацию магнитного поля в области датчика. Действие концентраторов сильно ослабевает при увеличении зазора между ними, поэтому его следует делать минимальным (равным толщине самого ДМТ). Усиление индукции концентраторами почти равно величине их магнитной проницаемости. В экспериментах при использовании стержней из мю - металла с зазором 0,3 мм удавалось получить увеличение чувствительности датчиков в 400 раз. Концентраторы магнитного поля позволяют улучшить соотношение сигнал - шум в 100 раз.

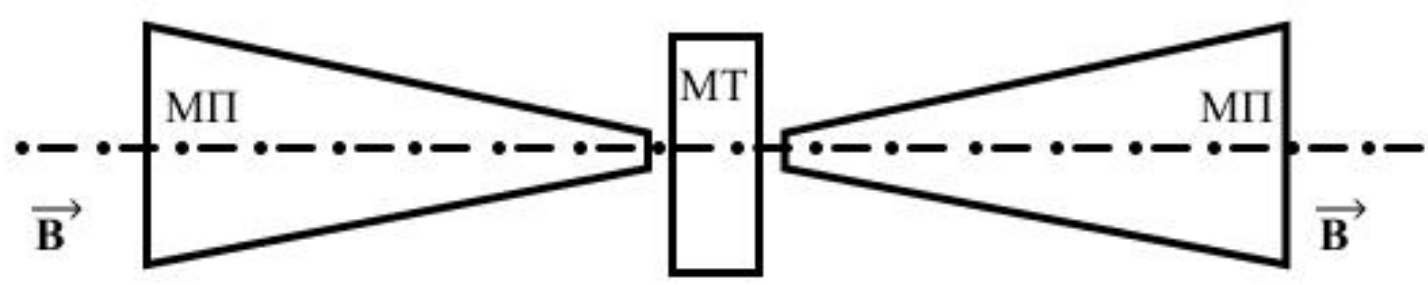

Рис.3. Расположение магнитопроводов относительно магнитотранзистора

Величина магнитного поля Земли в зависимости от широты местоположения составляет $25 \ldots 50 \mathrm{~A} / \mathrm{M}$, а создаваемая им магнитная индукция $0,3 \ldots 0,6^{*} 10^{-4}$ Тл. Если использовать для измерения ДМТ с концентраторами поля, усиливающими в 100 раз, то величина выходного сигнала составляет около 0,2 В. Поскольку полярный угол выходного напряжения изменяется в пределах $90^{\circ}$ (рис. 2), то угловая чувствительность составит 0,2 мВ/град.

Как видно из рис.1-б, с ростом температуры магниточувствительность ДМТ уменьшается. Для устранения этого недостатка использован дифференциальный усилитель из двух обычных транзисторов, коэффициент усиления которого растёт с увеличением температуры. В такой схеме уменьшение сигнала с ДМТ с ростом температуры компенсируется ростом коэффициента усиления усилителя, в результате чего на выходе усилителя удается получить сигнал, зависящий только от напряженности магнитного поля $B$ и практически не зависящий от температуры [2].

В экспериментальной конструкции электронного компаса использовалась микросхема, в которой ДМТ, транзисторы усилителя и резисторы создавались в одном кристалле кремния обычным методом двойной диффузии. Выходной сигнал такой микросхемы в диапазоне температур $0-40^{\circ} \mathrm{C}$ в пределах $5 \%$ точности измерений не зависел от температуры. Выходной сигнал микросхемы измерялся цифровым вольтметром. Как следует из рис. 2, каждому значению напряжения соответствует два значения угла. Для того, чтобы их различать, используется вторая микросхема с магнитотранзистором ДМТ-2, плоскость которого перпендикулярна плоскости первого. Зависимость выходного напряжения от угла для ДМТ-2 будет сдвинута на 90 относительно зависимости рис. 2. Пусть, например, ДМТ-1 показывает положительное напряжение. Тогда, если ДМТ-2 показывает отрицательное напряжение, то искомый угол находится в пределах $0 . . .90^{\circ}$, если же он показывает положительное напряжение, то угол находится в пределах $90^{\circ} . .180^{\circ}$. Подобным же образом определяются углы в пределах $180^{\circ} . .360^{\circ}$. 
Обычный компас, в его наиболее распространённом применении, есть прибор, показывающий направление на Север и на Юг. При использовании для этой цепи магнитотранзистора его удобно ориентировать так, как показано на рис. 1. В этом случае максимальное напряжение одной полярности будет соответствовать направлению оси магнитотранзистора (перпендикулярно плоскости базы) на Север, а максимальное напряжение противоположной полярности - на Юг. Однако, при отклонении оси от этого направления и на Запад, и на Восток напряжение уменьшается одинаково в обе стороны. Поэтому для определения направления отклонения также необходим второй магнитотранзистор, расположенный перпендикулярно первому (рис. 4). Тогда, например, при ориентации оси компаса на Север, ДМТ-1 показывает максимальное напряжение, а ДМТ-2 - нуль. При отклонении оси на Запад ДМТ-2 показывает напряжение одной полярности, а на Восток - противоположной. Напряжение с ДМТ-1 в обоих случаях уменьшается. Величина угла отклонения может отсчитываться либо по уменьшению напряжения от максимального у ДМТ-1, либо по отклонению напряжения от нуля у ДМТ-2.

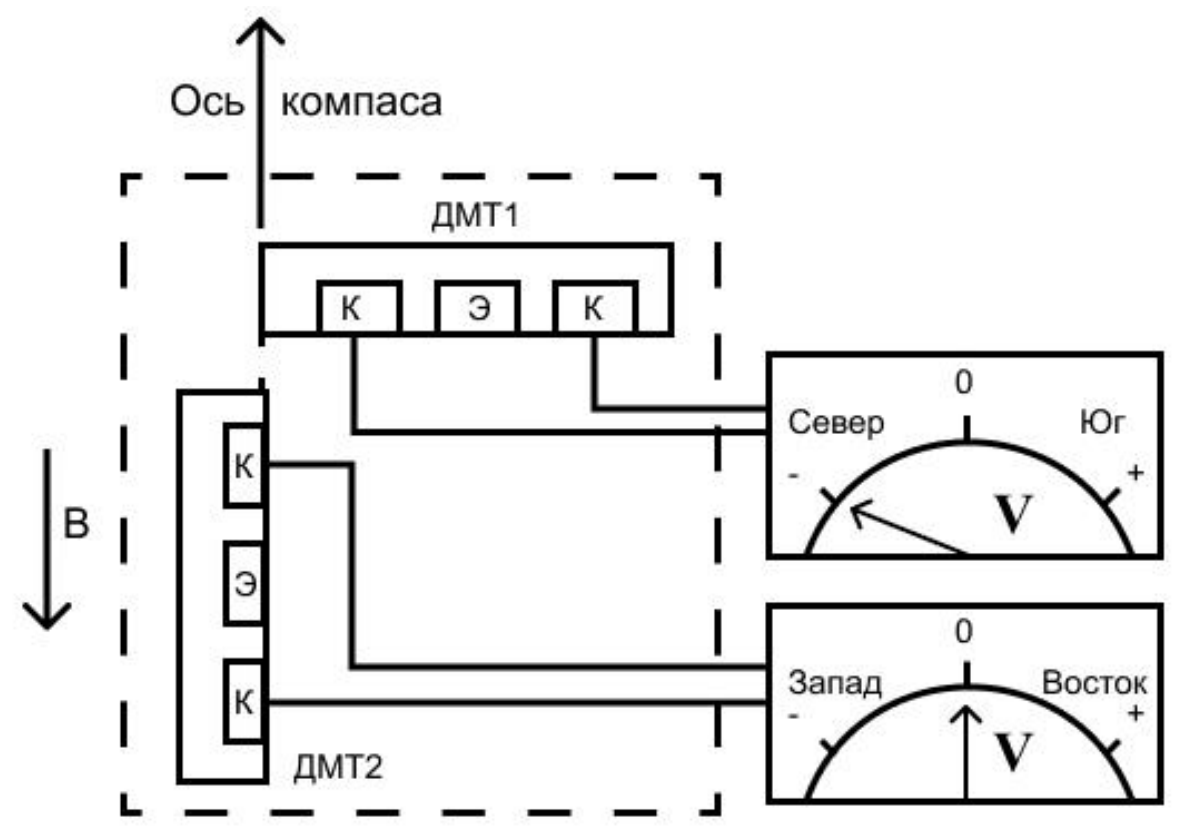

Рис.4. Расположение двух ДМТ в электронном компасе (вид сверху по отношению к рис.2)

Магнитотранзистор ДМТ может также использоваться и в качестве элемента, управляющего курсом движущего устройства, например морского судна. Установив ДМТ (линия, соединяющая базы) в заданном направлении движения, изменением нагрузочных сопротивлений устанавливается нуль напряжения между коллекторами. При отклонении оси от заданного направления в одну сторону, между коллекторами появляется напряжение одной полярности, а в другую - противоположной. Это напряжение через систему авторулевого может непосредственно управлять рулевым механизмом и автоматически выдерживать заданное направление движения морского судна.

Таким образом, экспериментально показано, что на основе двухколлекторного магнитотранзистора может быть создан электронный компас, не содержащий механически перемещающихся деталей, что резко повышает его надёжность и прочность.

Датчиком с использованием планарной конструкции ДМТ можно измерить только одну составляющую магнитного поля. В электронном компасе для определённых применений может потребоваться измерение всех трёх составляющих вектора магнитной индукции. С этой целью дополнительно также предложена и разработана конструкция магнитотранзистора кубической структуры (рис. 5), в которой эмиттер и контакт к базе 
расположены на противоположных по диагонали вершинах куба, а коллекторы на трёх соседних с эмиттером вершинах.

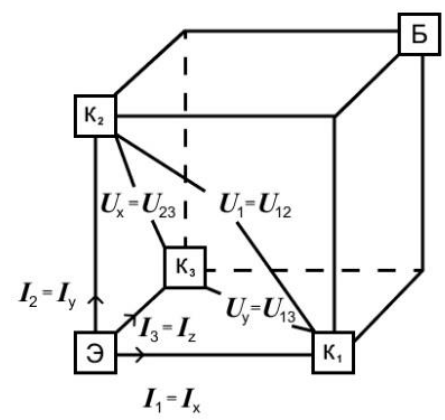

a)

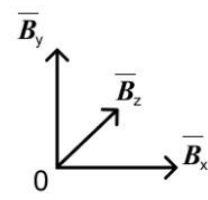

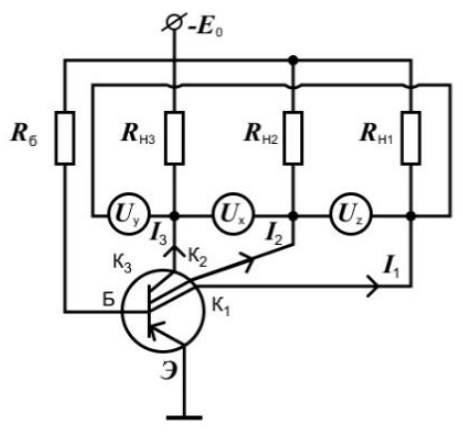

б)

Рис.5. Структура трехколлекторного магнитотранзистора а) и схема его включения б)

Магнитотранзистор работает следующим образом. При включении по схеме рис. 5-б при одинаковых резисторах $R_{1}-R_{3}$ в отсутствие магнитного поля поток инжектированных из эмиттера носителей разделяется на три равных потока $I_{x}=I_{1}, I_{y}=I_{2}, I_{z}=I_{3}$ в коллекторы $K_{1}, K_{2}$ и $K_{3}$. Вследствие равенства токов коллекторов равны и их потенциалы, а разность потенциалов между двумя любыми коллекторами равна нулю. При наличии магнитного поля на потоки носителей действует сила Лоренца, изменяющая их траектории. Пусть, например, вектор $\overrightarrow{B_{z}}$ направлен по оси $z$, т.е. $B_{z}=B$. В этом случае сила Лоренца действует только на два тока $I_{y}$ и $I_{x}$, направленные перпендикулярно направлению вектора $\overrightarrow{B_{z}}$. Поток $I_{y}$ прижимается этой силой к грани «у0х», при этом увеличивается скорость поверхностной рекомбинации на грани, и количество носителей, попадающих в коллектор $K_{2}$, уменьшается. Однако, это уменьшение компенсируется тем, что поток $I_{x}$ отклоняется от оси «0х» к оси «0у» и часть носителей этого потока попадает в коллектор $K_{2}$, вследствие чего его ток (и потенциал) практически не меняется. Ток же коллектора $K_{1}$ уменьшается, что приводит к увеличению его потенциала (за счёт уменьшения падения напряжения на резисторе $\left.R_{1}: U_{1}=E-I_{x} R_{1}\right)$. Таким образом, напряжение $U_{1}$ на коллекторе $K_{1}$ растёт с увеличением составляющей $B_{z}$ вектора магнитной индукции $\vec{B}$, и его можно записать как $U_{z}=U_{1}=f\left(B_{z}\right)$

Так как $U_{2}$ в этом случае практически не меняется, то разность потенциала между коллекторами $U_{12} \approx U_{1}$. Поэтому величину $\vec{B}$ можно определить как по измерению $U_{1}$, так и по измерению разности $U_{12}$, т.е. вольтметр $U_{z}$ можно включить между коллекторами $K_{1}-K_{2}$ Более того, измерение разности потенциалов между коллекторами $U_{12}$ более удобно, так как её отсчет (при $B=0$ ) ведётся от 0 , а потенциал $U_{1}$ измеряется от некоторой фиксированной величины, поэтому лучше принять $U_{z}=U_{12}=f\left(B_{z}\right)$

Аналогичными рассуждениями можно показать, что $U_{x}=U_{23}=f\left(B_{x}\right)$ и $U_{y}=U_{13}=f\left(B_{y}\right)$.

Следовательно, проградуировав шкалу каждого вольтметра непосредственно в единицах магнитной индукции, можно по их показаниям измерять величины всех трёх составляющих вектора магнитной индукции. 


\section{Выводы и перспектива дальнейшей работы по данному направлению}

Описаны принцип действия, физические процессы, схемотехническое решение и разработка конструкции принципиально нового электронного устройства, регистрирующего магнитное поле Земли и точно указывающее реальное направление по странам света. Данное электронное устройство не имеет подвижных механических частей и механизмов.

Предложено увеличить чувствительность датчика магнитного поля на основе двухколлекторного магнитотранзистора путём применения концентраторов магнитного поля. В результате экспериментов удалось получить увеличение чувствительности датчиков в 400 раз. Концентраторы магнитного поля также позволяют улучшить соотношение сигнал - шум на выходе электронной схемы в 100 раз.

Предложена схема дифференциального усилителя на полупроводниковых элементах, в которой уменьшение сигнала с двухколлекторного магнитотранзистора с ростом температуры компенсируется ростом коэффициента усиления усилителя, в результате чего на выходе электронной схемы удается получить сигнал, линейно зависящий только от напряженности магнитного поля $B$ и практически не зависящий от температуры.

Также предложено использовать схему на основе двухколлекторного магнитотранзистора и в качестве элемента, управляющего курсом движущего устройства, в частности морского судна. Выходной сигнал электронной схемы через систему авторулевого может непосредственно управлять рулевым механизмом и автоматически выдерживать заданное направление движения морского судна.

В электронном компасе датчиком с использованием планарной конструкции двухколлекторного магнитотранзистора можно измерить только одну составляющую магнитного поля. Вместе с тем, для определённых применений может потребоваться измерение всех трёх составляющих вектора магнитной индукции. С этой целью дополнительно также предложена и разработана конструкция магнитотранзистора кубической структуры. В этой случае разработанное устройство сможет выполнять функции только не только компаса, но и измерителя крена и дифферента.

Совершенно очевидно, что электронный компас станет обязательным атрибутом будущих автономных судов без экипажа. Он сможет не только заменить классический магнитный компас, но и позволить реализовать принципиально новые функции управления судном.

\section{ЛИТЕРАТУРА}

1. Викулин И.М., Викулина Л.Ф., Стафеев В.И. Гальваномагнитные приборы. - М.: Радио и связь, 1983. - 105 с.

2. Викулина Л.Ф. Схемное решение термостабилизации выходного сигнала полупроводниковых датчиков. // Технология и конструирование в электронной аппаратуре. - 1998. - №3-4. - С. 52-53.

3. Викулин И.М., Викулина Л.Ф., Горбачев В.Э. Магниточувствительные приборы для сенсорных и исполнительных сетей. - М.: РУСАИНС, 2019. - 156 с.

4. Викулин И.М., Викулина Л.Ф., Горбачев В.Э., Михайлов Н.С. Полупроводниковые инжекционные сенсоры магнитного поля комбинированного типа для беспроводных информационных сетей. // Известия ВУЗов: Радиоэлектроника. - НТУУ «КПИ им. И.Сикорского». - 2020. - Том 63, № 7 (697). - С. 437-447.

5. I.M. Vikulin, L.F. Vikulina, V.E. Gorbachev, N.S. Mikhailov. Combined semiconductor injection magnetic field sensors for wireless information networks. // Radioelectronics and Communication Systems, 2020, Vol. 63, № 7, pp. 368-375. - Allerton Press. - N.-Y. - 2020.

6. Nick Lemon. E-Nav advances. // Seaways: The International Journal of the Nautical Institute/ July, 2015. - London - UK: Stephens\&George, Merthyr Tydfil, 2015.- № 7. - p. 10 - 11. 
7. Petersen J.R., Eriksson O.F. Putting the mariner in the picture. // The Navigator: Aids to navigation . - 2015, No. 10, p. 4 -5.

8. David Patraiko. Cyber security on board. // Seaways: The International Journal of The Nautical Institute/ September, 2014. - London - UK.: Stephens\&George, Merthyr Tydfil, 2011. - p.9.

9. Paul Thomas. Cyber: it's about operational risk management. // Alert! The International Maritime Human Element Bulletin / Issue №39 September 2015. - London - UK. - p.6.

10. Sea Review: The International Maritime Journal of the Nautical Institute of Ukraine/ March, 2015. - Odessa - Ukraine.: p.40.

11. Wake Philip. Guarding against failure. // Seaways: The International Journal of The Nautical Institute/ September, 2014. - London - UK.: Stephens\&George, Merthyr Tydfil, 2011. - p.3. 